\title{
EWSR1/DDIT3 Fusion Protein
}

National Cancer Institute

\section{Source}

National Cancer Institute. EWSR1/DDIT3 Fusion Protein. NCI Thesaurus. Code C99201.

A fusion protein encoded by the EWSR1/DDIT3 fusion gene. This protein is comprised of the transactivation domain of the RNA-binding protein EWS followed by the DNA-binding basic domain and leucine zipper dimerization domain of the DNA damage-inducible transcript 3 protein. 